\title{
COMPETITIVIDAD SOSTENIBLE DE LA PEQUEÑA EMPRESA: Un modelo de promoción de capacidades endógenas para promover ventajas competitivas sostenibles y alta productividad
}

\author{
Braulio Vargas \\ Universidad ESAN \\ bvargas@esan.edu.pe \\ Carlos del Castillo \\ Universidad ESAN \\ cdelcastillo@esan.edu.pe
}

\begin{abstract}
Resumen
En las últimas tres décadas, la competitividad y la productividad de la pequeña empresa han merecido importantes esfuerzos de promoción, fomento y asistencia técnica del gobierno, la empresa y la cooperación técnica. En las economías en vías de desarrollo de la región Asia Pacífico estos esfuerzos se sustentan en el entendido de que la pequeña empresa aporta decisivamente a la creación de empleo, la dinámica económica local, la eficiencia colectiva del tejido empresarial y el crecimiento económico. El grueso de intervenciones ha sido canalizado a través de políticas públicas con un enfoque de construcción de capacidades. No obstante, estudios preliminares en los principales clusters empresariales en el Perú sugieren que las iniciativas de fomento concentradas en el accionar gubernamental no han tenido un efecto sostenido en la competitividad ni la productividad. La evidencia sugiere que los servicios de desarrollo empresarial tienen incidencia limitada porque dependen solo de intervenciones externas, a diferencia de los emprendimientos exitosos, que se explican mejor por capacidades endógenas. Este artículo introduce un framework de promoción de la productividad y la competitividad sostenida en la pequeña empresa, con énfasis en las capacidades endógenas y una combinación eficiente de intervenciones externas.
\end{abstract}

Palabras clave: competitividad, pequeña empresa, innovación, desarrollo sostenible, promoción empresarial.

\begin{abstract}
In the past three decades, the competitiveness and productivity of the small enterprise have been favored by the government, business action and technical cooperation, which have made significant contributions regarding promotion, development and provision of technical assistance. In the developing economies of the Asia Pacific region, these efforts are explained by the fact that the small enterprise contributes without question, to job generation, local economic dynamics, collective efficiency of business network, and economic growth. In the economies of the region, most contributions have been channeled through public policies with a focus on capacity building. Nevertheless, according to
\end{abstract}


preliminary studies in the main business clusters in Peru, the government's promotion actions have not had a steady effect in competitiveness and productivity. Evidence suggests that business development services have a limited effect as they depend solely on external interventions. In contrast, successful entrepreneurship relies more on endogenous capacities. This article introduces a framework of promotion of the productivity and sustained competitiveness in the small enterprise, laying stress on endogenous capacities and an efficient arrangement of external interventions.

Key words: competitiveness, small enterprise, innovation, sustained development, business promotion.

\section{Enfoques agregados en el fomento de la competitividad de las mypes}

Las pequeñas y microempresas (mypes), sobre todo las pequeñas, tienen una gran importancia para el crecimiento económico y la generación de fuentes de empleo productivo, con múltiples beneficios para la economía local, la eficiencia colectiva del tejido empresarial de la economía y la sociedad en general, como se ha demostrado ampliamente en estudios conducidos en todo el mundo. En el caso del Perú, este artículo llega a iguales conclusiones. No obstante, también ha identificado evidencia que lleva a sugerir que los beneficios prometidos se mantienen si es que las pequeñas empresas, en especial aquellas de sectores manufactureros, logran niveles sostenidos de competitividad y ganancias en productividad.

En el ámbito de las economías en vías de desarrollo del Sudeste Asiático y América Latina la visión de las mypes ha cambiado en los últimos 20 años. Se ha pasado de verlas como medio de sobrevivencia y fuente de informalidad a entenderlas como componentes clave en los procesos de desarrollo económico local y revitalización de la estructura productiva de un país o una economía.

Con esta óptica, los gobiernos se han constituido en las principales instancias promotoras de programas y esfuerzos público-privados para apoyar el crecimiento de la pequeña empresa a través de diversas estrategias como servicios de desarrollo empresarial, capacitación, microcréditos y asociaciones en torno a parques industriales, entre otros. No obstante, se ha encontrado un desbalance en cuanto a identificar, por un lado, en qué punto del «contínuum» de crecimiento y bajo qué condiciones tienen éxito en el mercado las mypes y, por otro, qué tipos de esfuerzo endógeno son necesarios para sostener el éxito empresarial en un contexto de negocios cada vez más turbulento.

Reconociendo las restricciones de recursos para el apoyo a las mypes en las economías en desarrollo, en años recientes ha surgido un interés creciente por entender qué factores son críticos para fomentar su competitividad. En un estadio inicial se ubican las estrategias de los gobiernos por invertir recursos públicos en la provisión de servicios de desarrollo empresarial e infraestructura que puedan ser aprovechados por los agentes económicos locales y los nuevos emprendimientos. En un estadio intermedio están las estrategias y los programas que buscan ofrecer a las mypes nuevas oportunidades de mercado al alentar la convergencia de su oferta de bienes y servicios con la demanda local y nacional; por ejemplo, a través de las compras estatales. En un estadio más 
avanzado se ubican los servicios más complejos de desarrollo empresarial, los proyectos y la asistencia técnica asociados a procesos de incubación de empresas, la financiación mediante capital de riesgo y los business angels, entre otros.

En los últimos 15 años, los tres estadios han sido adoptados de modo consistente en economías emergentes y en vías de desarrollo. Los resultados, en términos de crecimiento del producto, índices de supervivencia, adquisición de mypes innovadoras por empresas más grandes y empleo, sugieren que una combinación eficiente de estos esfuerzos y estrategias aumenta su nivel de incidencia. En otras palabras, en la medida que el apoyo sea integral, los indicadores agregados de evaluación de impacto de las inversiones realizadas tienden a aumentar.

También se ha identificado que el uso de indicadores agregados puede enmascarar diferencias sustanciales en términos de éxito empresarial e impacto en la economía local. Una apreciación de estas dos variables permite diferenciar dos tipos de emprendimientos. En un primer grupo, un número relativamente escaso de empresas, sobre todo pequeñas, destaca en el mercado y desarrolla innovaciones de productos, procesos, tecnologías de gestión y expansión a mercados externos. En un segundo grupo, un número mayoritario de unidades económicas, en especial pequeñas y microempresas de servicios y comercialización, no desarrollan innovaciones pues solo se ajustan a oportunidades coyunturales de mercado o fórmulas ya ensayadas por empresas más dinámicas y/o innovadoras; es decir, siguen aquello que ya funcionó para las empresas más exitosas.
Dentro de este marco, se plantea que las políticas y las estrategias convencionales de fomento a las mypes no parecen contribuir a niveles sostenidos de competitividad. Tampoco parecen explicar qué otros factores son relevantes para que los indicadores individuales, por sector productivo y empresa, tengan un comportamiento positivo y homogéneo que haga más confiables los indicadores agregados. Este razonamiento sugiere una rama de estudio que llevaría a un mejor entendimiento de aspectos endógenos como la acumulación acelerada de capital, el mayor acceso a financiamiento formal a tasas que se aproximan progresivamente a aquellas de las cuales disfrutan las empresas medianas, el desarrollo de nuevos productos, el empleo de mejor tecnología, la obtención de clientes de mayor tamaño y el acceso a mercados exportadores, entre otros aspectos, muchos de los cuales están asociados a la noción de «competencia imperfecta».

Una de las corrientes de pensamiento más citadas y que parece explicar bastante bien cuál es el factor faltante en la fórmula del éxito y la competencia imperfecta es el emprendimiento (entrepreneurship); el cual, en el ámbito de las mypes, asocia el éxito empresarial a un tipo de agentes económico-sociales que tienen una motivación intrínseca para llevar adelante sus ideas de nuevos negocios, productos, procesos, mercados, formas de aplicar las estrategias desarrolladas por otros, etcétera.

No obstante, el estudio del emprendimiento no ha logrado aún asociar el éxito de algunos emprendedores solo con el factor de motivación intrínseca. Otros elementos que gravitan en torno al éxito económico y el nivel de competencia también deben alcanzar a las pequeñas empresas sobre la base de una combinación eficiente de 
buen precio, buen producto, altos niveles de productividad y buena gerencia. Por tanto, las nuevas orientaciones en el estudio y el diseño de políticas de fomento requieren dirigirse a identificar cuáles son los factores asociados a la competitividad y la productividad que explican o pueden explicar el éxito económico de las pequeñas empresas.

En este sentido, se hace más clara la necesidad de que los esfuerzos gubernamentales basen el diseño de sus políticas de fomento en intervenciones externas que, por un lado, se apoyen en el sector empresarial más consolidado y, por el otro, en pequeñas empresas que asuman el reto y el compromiso de participar de procesos de desarrollo económico local e inversión en sus territorios. Subsecuentemente, una combinación de intervenciones externas e internas amplía la incidencia de los esfuerzos en favor de la competitividad sostenida y mayores niveles de productividad en la microempresa y especialmente en la pequeña empresa.

\section{Elementos de competitividad externa de las pequeñas empresas}

En este artículo se define la competitividad externa de la pequeña empresa como la capacidad creciente de aprovechar, dadas ciertas condiciones de competitividad sistémica en el entorno, las oportunidades de mercado y obtener un buen desempeño empresarial, en términos de ganar aceptación y participación de mercado sobre la base de buena información, procesos, productos, precios, servicios, estrategias de mercadeo y servicios posventa.

La competitividad externa alude a los tipos de apoyo brindados por un agente externo que se ponen a disposición de una masa crítica de mypes para mejorar sus procesos, oportunidades de acceso a infraestructura de soporte para las operaciones productivas y la comercialización, y sistemas de toma de decisiones administrativas, productivas, de mercadeo y relación con los clientes.

Sobre la base de una sistematización de los tipos de intervenciones externas más frecuentes para que las mypes mejoren su competitividad, en el gráfico 1 se muestra cuatro pilares críticos para ayudar elevar su competitividad externa.

- Eje de productividad: Recursos, incluidos recursos humanos y financieros, $\mathrm{y}$ procesos.

- Eje de competitividad: Desarrollo de buen producto y acceso a mercados.

Destaca que la mayoría de intervenciones, sobre todo desde el Gobierno Central o los gobiernos locales, las ONG y las entidades de microfinanciamiento y capacitación, se ha concentrado en el eje de la competitividad; específicamente en los factores asociados al acceso a información sobre el mercado, la asociatividad y la capacitación en temas gerenciales; por ejemplo, márketing. Esto ha hecho que se ponga menor énfasis en otros factores que apuntalan el eje de productividad y que van más allá de los decretos y las políticas públicas, los cuales descansan en un marco normativo. Por el contrario, estos factores asociados a la productividad se apoyan en el acceso a nuevas tecnologías productivas y de gestión, la capacidad gerencial con visión emprendedora, la mejora en las condiciones laborales y el desarrollo o la adopción de buenas prácticas de negocio y responsabilidad social. 


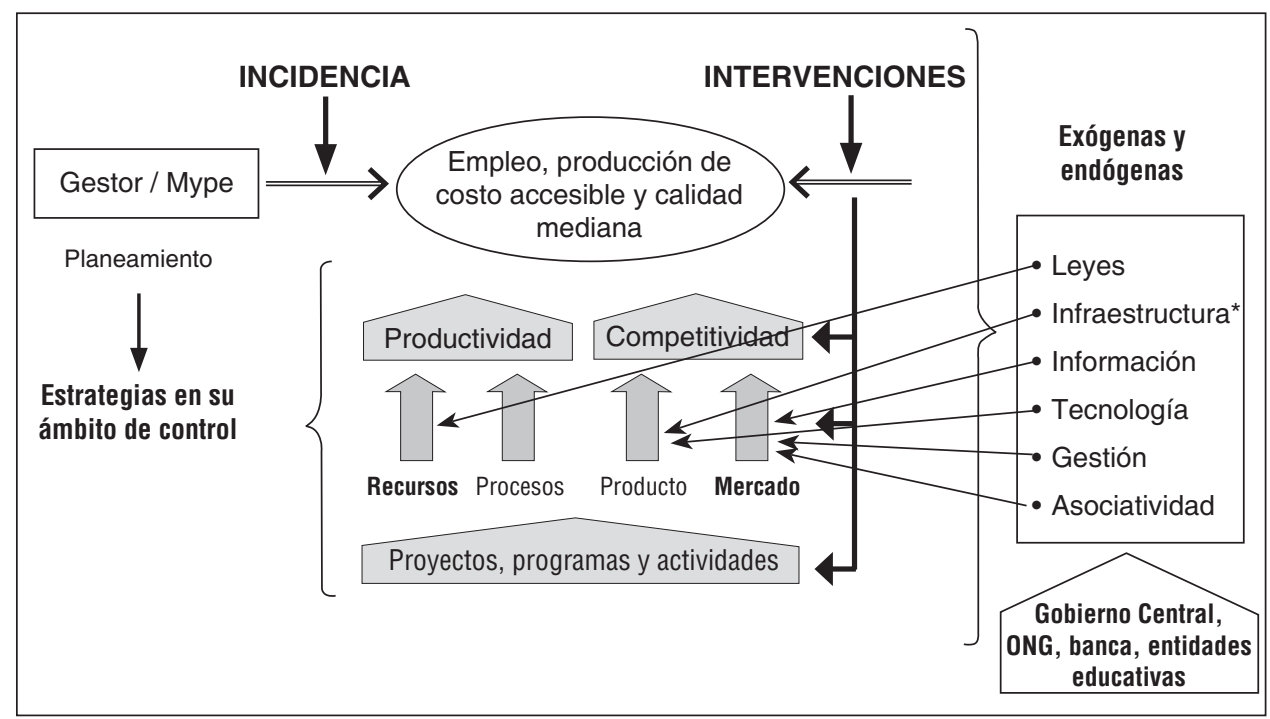

* Incluido el acceso a fuentes de financiamiento y/o microcréditos.

\section{Gráfico 1}

Entorno de competitividad externa de las mypes

Los determinantes de la competitividad externa se agrupan en dos grandes grupos: el primero comprende la creación de condiciones y la oferta de servicios de asistencia para el desarrollo empresarial y el segundo consiste en el conjunto de capacidades que deben reflejar la madurez empresarial del emprendedor y la empresa.

\subsection{Intervenciones exógenas}

Para entender el aporte de este primer grupo de intervenciones es necesario tener en cuenta el tipo de problemas o restricciones a los que responden. Esto es posible a través de una rápida revisión de la problemática de la pequeña empresa.

En términos generales, en las economías en desarrollo, las mypes surgen en su mayor parte debido a la existencia de una brecha entre la oferta y la demanda de empleo. El número de grandes empresas con capacidad de generar altos volúmenes de empleo es limitado; más aún, lejos de aumentar, en muchos casos esta capacidad se ha reducido por el avance científico y tecnológico que, en los últimos 50 años, ha vuelto obsoletos muchos puestos de trabajo. Una aproximación económica permite entender que esta menor demanda de creación de puestos de trabajo se explica por la concentración de los nuevos puestos de trabajo en aquellos roles que requieren más conocimiento $\mathrm{y}$, por tanto, personal mediana y altamente calificado.

Hasta hace tan solo tres décadas, los trabajos calificados de más alta demanda se asociaban a labores repetitivas; por ejemplo, operar comandos que activaban una línea de manufactura, realizar tareas como bajar y subir palancas, operar una máquina-herramienta, etcétera. En estos casos, la mano de obra rutinaria y «el buen ojo» eran instrumentos de precisión útiles para 
operar y controlar el flujo de los procesos. En cambio, en los procesos productivos actuales las labores se han automatizado progresivamente $\mathrm{y}$, en muchos casos, el desempeño de los operarios lo realiza ahora un computador. Estos equipos y programas de computadora son más precisos y veloces, no se agotan ni pierden concentración como ocurre cuando alguien tiene que efectuar la misma operación todos los días en forma rutinaria. En consecuencia, se ha reemplazado la mano de obra de baja calificación.

Por otro lado, los puestos de trabajo cuya demanda aumenta son aquellos que exigen un tipo de «capacidades» que no se puedan automatizar, o cuya automatización sería muy costosa. También incluyen aquellas funciones en las cuales la creatividad, el sentido común y el razonamiento crítico aportan a la calidad, la mejora en la toma de decisiones y la innovación de los procesos, entre otros aspectos intangibles que explican la rentabilidad de las empresas en el actual contexto de negocios. En definitiva, los trabajos de mayor complejidad que suelen demandar las empresas medianas y grandes no están al alcance de un gran número de ciudadanos $\mathrm{y}$, en general, de emprendedores que no tienen la calificación necesaria. Lo que no niega que puedan poseer los talentos asociados a la innovación, la creatividad y el compromiso con los resultados de la empresa, factores que inciden tanto en la competitividad sostenida como en ganancias de productividad.

Paradójicamente, el mismo fenómeno de reemplazo de la mano de obra por las ventajas de la tecnología alienta el surgimiento de los emprendimientos locales, en especial en las economías en vías de desarrollo caracterizadas por niveles elevados de desempleo y pobreza. Este fenómeno se comprueba al ver que los países que figuran en los primeros sitios del ranking de emprendedores del reporte Global Entrepreneurship Monitor son países en vías de desarrollo como el Perú. De modo general, también se puede afirmar que el deseo de sobrevivir y generar autoempleo despierta la creatividad de los emprendedores y el trasfondo familiar que suele caracterizar al proceso de surgimiento de las microempresas y, posteriormente, las pequeñas empresas.

En las mypes familiares la fuerza de trabajo productivo de relativamente baja calificación está contenida en el emprendedor. Eventualmente, los familiares cercanos se adhieren a la visión del emprendedor y se crean las primeras posiciones de trabajo de mediana especialización (operarios para nuevos procesos, funcionarios para afrontar las necesidades de gestión del negocio, etcétera) y los primeros códigos de conducta o valores en la firma, como ahorro, capitalización, reinversión, desarrollo de políticas de adquisiciones y pago a proveedores y personal. Esta transición ha determinado un viraje de la actividad microempresarial centrada en la producción o la prestación de servicios «bajo demanda» o «bajo condiciones de aprovechar una coyuntura de mercado», hacia la búsqueda de eficiencia y ganancias en productividad y competitividad. La evidencia más frecuente de esta transición se puede apreciar en que el primer tipo de financiamiento obtenido por las pequeñas empresas no proviene de entidades especializadas si no que es brindado por los proveedores en respuesta a «la confianza».

Así, por ejemplo, este estudio ha identificado que los proveedores eventualmente brindan a las mypes las mismas condiciones de crédito que suelen brindar a firmas 
de mayor tamaño: compras a pagar en 30 días o más y tasas de interés más bajas que las que podrían obtener si se financiasen a título personal o si se solicitase crédito a la banca formal. En síntesis, la creación y el crecimiento de las mypes tienen su origen en la combinación de un espíritu emprendedor y las necesidades de bienestar de las familias. Esta combinación resulta un germen imprescindible para que tengan un alto impacto en la dinámica económica local y la creación de empleo. Por tanto, deben ser la base de gran parte de los esfuerzos del sector gubernamental (sobre todo de los gobiernos locales) para promover el crecimiento, la competitividad y la productividad de este estrato empresarial.

Dentro de este razonamiento, las medidas convencionales de fomento, particularmente desde el sector gubernamental, consiguen un nivel externo de incidencia cuyo efecto parcial no llega a fusionarse con los factores endógenos que caracterizan la evolución del emprendedor y el crecimiento de la unidad económica familiar. Elementos que van de una visión o una respuesta a las necesidades humanas hasta una unidad económica que enfatiza la eficiencia, la productividad, la expansión de mercados y la innovación de procesos y productos, entre otros. No obstante, las intervenciones externas han demostrado ser necesarias pero insuficientes y se han traducido en iniciativas de fomento como:

- Servicios de desarrollo empresarial: Orientados a la capacitación de pequeños y microempresarios, acceso a fuentes de información de mercado, calificación de la mano de obra en procesos industriales de pequeña escala, constitución de empresas y formalización de las existentes, etcétera.
- Inversión en infraestructura básica: Para facilitar un mejor acceso al mercado y añadir eficiencia a los procesos productivos. En este rubro destacan los maquicentros o centros que brindan servicios de manufactura en una especialidad, como torneado de piezas metálicas; los centros de innovación sectoriales orientados a desarrollar innovaciones que luego sean puestas al servicio de los gremios de pequeños empresarios en sectores productivos de baja complejidad, como calzado, metal-mecánica, artesanía o confecciones; la construcción de caminos y puentes para mejorar el acceso al mercado; la dotación de los servicios de energía y saneamiento necesarios para producir; etcétera.

- Microcréditos: Esta iniciativa ha resultado ser un pilar importante para la supervivencia y crecimiento de las mypes. Los préstamos, inclusive cuando son caros con tasas de interés mayores al promedio de la banca formal, son menores a las tasas de las fuentes informales que pueden llegar a un 100\% mensual. El microcrédito ha servido como incentivo externo para que los pequeños empresarios se vean alentados a invertir en innovaciones, mejora de infraestructura productiva, merchandising para atraer nuevos clientes, en especial medianas empresas, y ordenen las finanzas de la empresa. También han servido como fuente de fondos para ampliar la capacidad instalada, lo que resulta clave para atender mayores niveles de demanda como los que caracterizan a las medianas y las grandes empresas, lo que favorece la subcontratación, y otros beneficios similares. 


\subsection{Intervenciones endógenas}

Este segundo grupo de intervenciones compete directamente a los emprendedores $\mathrm{y}$, en menor medida, a los organismos competentes en materia de desarrollo económico local y fomento a las mypes. Estas intervenciones se orientan a mejorar la gestión empresarial, la estandarización y la normalización de procesos, la adopción de nuevas tecnologías y, aunque de modo incipiente, la integración de las mypes en los sistemas de innovación locales.

Un aspecto que destaca en este tipo de intervenciones es la gestión empresarial. Es el que se impulsa con mayor frecuencia y el que, en cierta forma, ha tenido resultados más visibles y permanentes. Esto último se debe a que la gestión empresarial es un proceso de aprendizaje y adopción de una cultura de negocios orientada al cliente y la obtención de resultados. Iniciativas como los bonos de capacitación han sido alternativas interesantes de transferencia de conocimiento en gestión que encuentran su correlato en los resultados del negocio, lo que determina que sean los mismos empresarios los que priorizan este tipo de asistencia en sus requerimientos de apoyo.

El medio más frecuente para estimular mejoras en la gestión empresarial ha sido la capacitación y el entrenamiento en temas como márketing, calidad total, formación de alianzas con los clientes, incluso con los competidores, y ubicación de pequeñas y microempresas en determinados distritos industriales que prometen, entre otros beneficios, ampliar el poder de negociación de las mypes.

En suma, las intervenciones endógenas desde una fuente externa también son necesarias pero no suficientes para impulsar los dos ejes centrales del éxito empresarial sostenido en las mypes. El gráfico 2 muestra los tipos de intervenciones que favorecen la competitividad y la productividad desde un punto de vista endógeno. Se aprecia que las medidas de fomento se concentran en las categorías mercado y producto. La poca diversidad de intervenciones en el entorno de las mypes coincide con el también escaso número de las instituciones que las ofrecen.

Una observación pertinente alude a la ausencia, excepto en seguridad social, de iniciativas en el campo de la mejora de las condiciones de trabajo como higiene y seguridad industrial, adopción y desarrollo de tecnologías de la producción, cálculo de costos de los procesos, reconfiguración de las líneas de productos, innovación de productos, inteligencia comercial y servicio al cliente.

Otra ausencia es la falta de sistemas de costos que permitan determinar qué actividades, tareas o procesos agregan valor económico, y qué aspectos implican actividades de soporte y están asociadas a gastos e inversiones que se deben automatizar u optimizar.

En cuanto a las medidas que apuntan a la mejora de la gestión empresarial, si bien tienen incidencia en el fomento de una cultura de negocios y negociaciones, no garantizan la competitividad sustentable ni la productividad creciente de las mypes debido a que disponer de más información no implica su aprovechamiento en aspectos como mayor creatividad o responsabilidad empresarial, o la internalización de códigos de negocios que privilegien las alianzas y los vínculos de negocio en una óptica «ganar-ganar». 

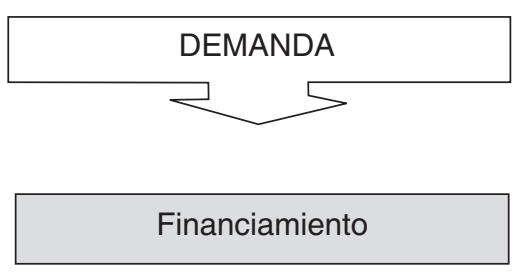

Capacitación
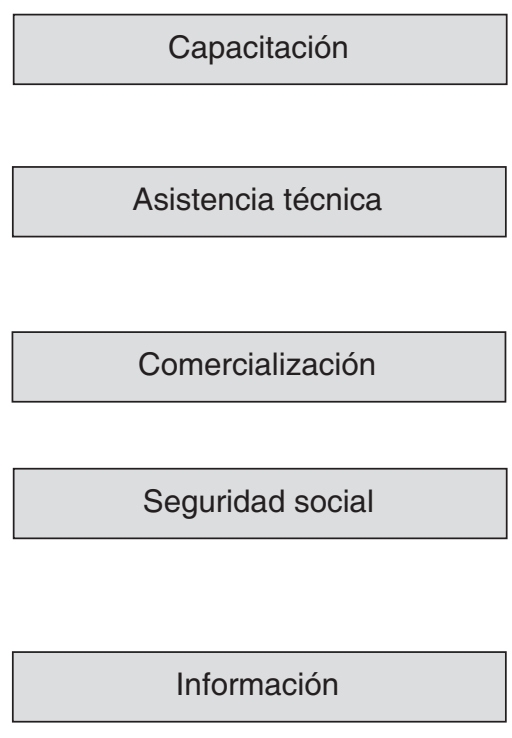

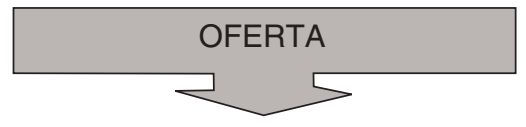

- Instituciones bancarias

- ONG

- Edpymes

- Instituciones públicas y privadas

- ONG y proyectos especiales

- Instituciones públicas y privadas

- ONG y proyectos especiales

- Consultores independientes

- Instituciones públicas y privadas

- ONG y proyectos especiales

- Consultores independientes

- Instituciones públicas y privadas

- Revistas especializadas

- Internet

- Centros de investigación

- Instituciones especializadas

- Asesores culturales

- Proveedores de bienes y servicios

Gráfico 2

Demanda y oferta de servicios de las mypes

Estos aspectos tienen mucha relación con los valores y la filosofía empresarial que adoptan las empresas que no solo desean ganar rentabilidad en el corto plazo, sino también en el mediano y el largo plazo. Esta evidencia alerta sobre la falta de nuevos tipos de protocolos del tipo «sociedadempresa» que muestren a los empresarios que distribuir los mayores rendimientos del negocio, mejorar las condiciones de trabajo y estar atentos a escuchar e intentar aplicar los aportes que puedan brindar los trabajadores, en especial los más experimentados, son factores críticos para el crecimiento de la pequeña empresa.

Mientras que para la gran mayoría de mypes se considera que invertir en mejorar el ambiente de trabajo y alentar un pacto de mutua solidaridad es un gasto o un lujo que las pequeñas empresas no se pueden permitir, las empresas más exitosas en el mercado sí parecen incorporar los factores satisfacción en el trabajo y fomento al trabajador como parte de su estrategia de crecimiento y sistema de gestión. En esta 
estrategia también se encuentran la innovación, la eficiencia operativa, la información de mercados, la calidad en el servicio al cliente y, de ser el caso, el grado de integración de la empresa al esquema del sistema nacional o local de innovación.

En particular, la integración multiplica las conexiones de la empresa innovadora con el resto de agentes económicos, sociales e institucionales en un territorio, lo que genera un importante impacto en la transformación de la realidad local. En este sentido, es la posibilidad de articular la pequeña empresa de alto rendimiento con el resto de los sistemas económicos, sociales e institucionales locales o nacionales el criterio estructural que determina la importancia de contar con políticas de fomento para la pequeña empresa (la causa), pues los beneficios (el efecto) de fomentar la pequeña empresa se traducen en indicadores de aumento del empleo, crecimiento del producto, incremento de recursos fiscales, subcontratación e impulso a la demanda interna y la asociatividad empresarial, entre otros.

El deslinde entre las intervenciones externas que se enfocan en el efecto (los beneficios de apoyar a las pequeñas empresas) y aquellas que se enfocan en la causa (el desarrollo de capacidades en la firma para crear conexiones con su entorno) permite llegar a conclusiones muy interesantes. Por un lado, tanto la atención en la causa como en el efecto son necesarios pero no suficientes por sí solos. Por otro lado, enfocarse en la causa, es decir, en la articulación de las mypes al sistema de innovación local y a otros sistemas presentes en el territorio, es complejo y alude a esfuerzos de gran envergadura que requieren de la intervención del sector gubernamental y, en muchos casos, también del sector empresarial. Lo que refuerza que los emprendimientos locales dependan del apoyo que agentes externos puedan brindar, desalentando el espíritu emprendedor y el deseo de ser más competitivos.

En consecuencia, en el ámbito territorial inmediato de actuación de las mypes, los espacios locales, los gobiernos tienen ante sí un desafío mayor respecto del apoyo a la pequeña empresa local. Si optan por políticas de fomento basadas en el efecto, su incidencia será parcial y estará restringida al corto plazo porque la competitividad de la pequeña empresa local depende en mayor proporción de factores brindados desde el sector externo, aunque este accionar es políticamente más redituable. Si optan por políticas basadas en la causa, la incidencia será integral y el impacto se extenderá en el largo plazo, lo que aporta al modelo de competitividad sostenida. Los resultados de largo plazo pueden desanimar la voluntad política. Además, la envergadura del esfuerzo, la exposición al largo plazo y el volumen de inversión de recursos y capacidades (curva de aprendizaje) requeridos, por ejemplo, para crear un sistema de innovación local, dificultan y ponen en riesgo la implementación de esta opción.

En cualquier caso, ninguna de las dos alternativas es suficiente por sí sola en términos de garantizar la implementación y amplificar el efecto y el impacto de las intervenciones. Al mismo tiempo, ambas opciones están expuestas a los riesgos de sustentar la competitividad de las pequeñas empresas en intervenciones externas; requieren de una curva de aprendizaje suficiente de los emprendedores y los agentes promotores; deben estar debidamente sustentadas en un marco normativo-jurídico que sea explícito en cuanto a que, por ejemplo, el gobierno local es el ente competente 
en materia de promoción del desarrollo económico local a través del fomento de la pequeña y microempresa.

\section{Elementos de la competitividad interna de las pequeñas empresas}

Una intervención que al mismo tiempo sea integral, sostenible y capitalice las fuerzas endógenas de emprendimiento requiere de un conjunto complementario de intervenciones de carácter interno en el cual el foco de atención sean las buenas prácticas empresariales que aporten a la competitividad, la eficiencia interna y la innovación para conseguir ganancias en productividad. Este esquema complementario se ha denominado modelo de competitividad interna.

Los estudios exploratorios conducidos para realizar este estudio sugieren que la dimensión interna que contribuye a aumentar sostenidamente el grado de competitividad de la firma está determinada por una combinación eficiente de gestión empresarial, visión y espíritu de emprendimiento. En la intersección de estas dimensiones se han identificado la innovación, la responsabilidad social y la noción de «distribución de los resultados del negocio». Cada uno de estos elementos ya ha demostrado su contribución al crecimiento, la imagen y la rentabilidad de los negocios en las empresas grandes y medianas; pero también resultan relevantes dentro de la fórmula identificada en las pequeñas empresas de éxito.

Estos tres aspectos internos constituyen el complemento eficiente de los factores de competitividad externa. Ejercen un mayor efecto multiplicador que las intervencio- nes convencionales, absorben bien los riesgos de exposición al largo plazo y los riesgos políticos, reducen la intensidad de recursos y capacidades necesarios para su implementación y son menos sensibles a los cambios en el contexto de las decisiones de los agentes gubernamentales y empresariales que suelen apoyar a la pequeña empresa. Lo más importante es que los factores de competitividad interna, que se muestran en el gráfico 3, ejercen una influencia directa sobre el eje de productividad, en particular en el uso de los recursos y la gestión de los procesos.

En el gráfico 3 se aprecia que la determinación de elementos o factores de competitividad interna tiene un efecto estabilizador dentro de la firma. Los elementos de competitividad interna inciden directamente tanto en el grado de competitividad de la firma como en la productividad y el uso de recursos, capacidades y procesos. En tal sentido, el modelo de competitividad interna absorbe las intervenciones externas e internas, es decir, los elementos de competitividad externa, y los emprendimientos internos.

El enfoque de competitividad sostenida que combina elementos de competitividad externa e interna aprovecha mejor las sinergias asociadas a la participación de los actores que son centrales en el impulso a la economía local, como gobiernos locales, gremios o asociaciones de pequeños y microempresarios y ciudadanos en general; y las de aquellos actores que forman parte de los esfuerzos de apoyo a la pequeña empresa como las ONG, agencias o programas especializados del Gobierno Central, las entidades financieras y el sector académico. Los elementos de competitividad interna se describen a continuación. 


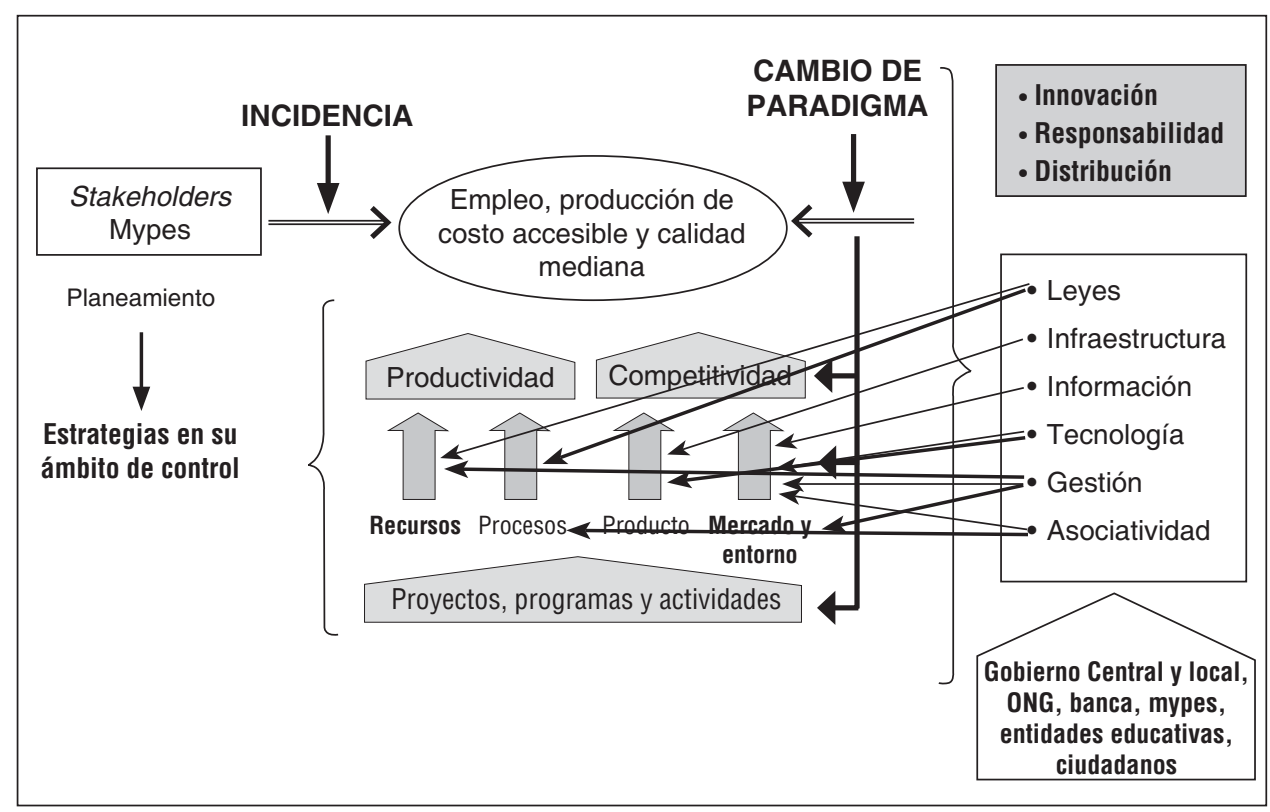

Gráfico 3

Entorno de competitividad interna de las mypes

\subsection{La innovación}

La creación de riqueza y bienestar es el fiel reflejo de la competitividad sustentable en un espacio territorial nacional o subnacional. El trasfondo de este proceso está regido por la innovación y el diseño de políticas para incentivar y sostener la creatividad de los emprendedores, generar condiciones propicias de desarrollo económico local y fomentar un mejor clima de negocios que sirva para atraer inversión productiva de alto valor, útil como catalizador del progreso técnico que alienta el uso económico de las innovaciones.

La literatura consultada refiere que la innovación es esencial para desarrollar los mercados de oferta y demanda de bienes y servicios. Por el lado de la demanda, los consumidores o los agentes que demandan bienes y servicios finales o intermedios son más propensos a utilizar y consumir nuevos productos en la medida que les atribuyen valor agregado o ventajas que los productos de uso convencional no consiguen equiparar. Por el lado de la oferta, los agentes económicos identifican ventanas de oportunidad en la innovación porque conduce al escenario schumpeteriano de la destrucción creativa, la competencia imperfecta y las ganancias extraordinarias (windfall profits), las cuales, al mismo tiempo, tiene un gran impacto sobre el progreso técnico, la transformación de las industrias, el recambio de las posiciones de liderazgo y la creación de nuevos nichos de mercado (disruptive innovation).

Los beneficios de la innovación han sido asociados más a la dinámica de la mediana y la gran empresa, particularmente 
en las economías más desarrolladas. Esto ha llevado a suponer que las mypes en economías en desarrollo como el Perú tienen mejores oportunidades de entender la innovación como un proceso de introducción de productos, procesos y tecnologías de gestión que resultan novedosos dentro de las fronteras nacionales pero que han sido originalmente desarrollados en otras regiones o países. No obstante, a partir del análisis de experiencias en el Perú, desde el año 2001, en especial en el distrito de Villa El Salvador, se ha identificado que la adopción por parte de emprendedores creativos de una fórmula que combina la innovación como eje estratégico del negocio y una cultura empresarial responsable conducente al compromiso y la redistribución de los resultados del negocio entre los stakeholders, principalmente trabajadores y operarios, moviliza el ciclo de retroalimentación positivo que impulsa la innovación de tipo emprendedor y, en el mediano plazo, la posibilidad de transición de la pequeña y microempresa hacia el estadio de mediana empresa.

La innovación emprendedora permite actualizar las tendencias de evolución de los mercados de oferta y demanda de bienes y servicios. Al ser inevitable, porque los clientes buscan nuevas formas de satisfacer sus necesidades actuales o tienen nuevas necesidades que satisfacer, los emprendimientos que se sustentan en la innovación destacan en sus sectores y desarrollan niveles de competitividad elevados, sin importar si se trata de una pequeña empresa. La innovación también conduce a la diversificación de la oferta, la discriminación de precios y el trabajo con nuevos segmentos de clientes.

Así, una mype innovadora puede diseñar distintas versiones de un producto, proponer distintos precios y combinar ambas estrategias en función del tamaño y la ubicación de sus clientes en el mercado. Será frecuente encontrar productos estándar, pues se espera que los productos «económicos» sean más accesibles $\mathrm{y}$, aunque ofrecen menor margen de utilidad, tengan alta demanda. Los ingresos que produzcan servirán para generar amplia información sobre la actividad regular de la empresa y para diluir los costos fijos y de operación. Existirán también productos diferenciados o de valor agregado que tendrán precios más altos $\mathrm{y}$, aunque se vendan en menor cantidad, ofrecerán altos márgenes de utilidad. Los ingresos que produzcan servirán para mejorar la imagen y el posicionamiento de la empresa y para financiar nuevas innovaciones, mejor infraestructura, mayor capacidad instalada y acumulación de activos (capitalización). Eventualmente, la solvencia económicofinanciera producto de la renta por productos estándar y diferenciados dará lugar a los productos mixtos. Estos consisten en la oferta de bienes o servicios de costo bajo, medio o alto; pero en realidad se tratará de productos que atienden demandas específicas o de nicho, cuyos ingresos servirán para incrementar el aprendizaje de la firma, aumentar su flexibilidad y capturar clientes leales atraídos por la capacidad de la firma de brindar bienes y servicios a la medida de sus necesidades.

Como resultado, la combinación de estos tres tipos de oferta permite ampliar la cuota de mercado, expandir la presencia del negocio o ingresar a nuevos mercados o nichos de mercado y mejorar la cartera de clientes. Esto implica que una mype innovadora estará en capacidad de vender a clientes pequeños, medianos y grandes. Sus ventas subirán, su rentabilidad se verá incrementada y adquirirá mayor poder de 
negociación. Cabe esperar que la diversificación de la oferta sea un indicador del grado de innovación que produce retornos reales crecientes (increasing returns) para la empresa; no habrá, por tanto, razón para dejar de innovar.

\subsection{La responsabilidad social empresarial}

Las empresas exitosas tradicionalmente han sido aquellas que sostienen su presencia en el mercado. Tanto la cantidad como la calidad de su oferta, ajustada a la demanda de mercado, han sido suficientes para permanecer; pero los cambios dramáticos en la tecnología y los mercados y el surgimiento de una mayor sensibilidad hacia aspectos de la realidad que se encuentran en el centro de atención de gobiernos y sociedades han alterado esta situación.

Aquellas empresas que no han incorporado estas nuevas metas no han sobrevivido o han perdido su posición anterior; lo que se puede verificar al comprobar que las empresas que lideraban los sectores relevantes de la economía hace tan solo dos décadas han cedido posiciones ante otras empresas en el mismo sector y nuevos competidores en sectores emergentes, como los sectores intensivos en conocimiento. Estos nuevos incentivos están asociados a la innovación, el conocimiento y la tecnología y la sensibilidad ante los nuevos desafíos que afronta la sociedad global; por ejemplo, los aspectos ambientales, la responsabilidad social empresarial y los regímenes laborales, entre otros.

Particularmente en las economías emergentes y en vías de desarrollo de la región Asia Pacífico existe aún cierto escepticismo con relación a abordar la responsabilidad social empresarial desde la perspectiva de las pequeñas y microempresas. En el mejor de los casos, las grandes empresas de Occidente son las que toman estos factores en cuenta. No obstante, en este estudios se ha encontrado evidencia de que las pequeñas empresas innovadoras exitosas y con mejor imagen en sus mercados han ampliado su liderazgo en virtud de hallar en los nuevos desafíos empresariales una oportunidad. Estas han combinado conocimiento, asociatividad y cooperación, sensibilidad social y sentido de pertenencia a la comunidad con sus stakeholders, sobre todo los trabajadores, para adoptar y mantener buenas prácticas de responsabilidad social como parte de su fórmula de éxito y crecimiento.

A continuación se desarrollan cuatro aspectos fundamentales que se deben considerar en el contexto de la responsabilidad social empresarial.

\section{La gestión del conocimiento}

Al observar los rankings de empresas, se puede apreciar que aquellas más exitosas en términos de ingresos, valor de mercado, capitalización y valor de activos trabajan en sectores e industrias muy dinámicos o tecnificados. Es frecuente que las primeras posiciones las ocupen empresas de alta tecnología, como Microsoft, Oracle, SAP, Apple y corporaciones que operan en una escala global sobre la base de procesos muy tecnificados como es el caso de los sectores energía, petroquímica y automovilístico.

Lo que estas empresas o corporaciones tienen en común es que lideran el avance científico y tecnológico y lo traducen en bienes o servicios tangibles a través de la innovación, el aprendizaje y la aplicación del conocimiento. Estos productos han cambiado la forma en que los humanos 
vivimos y generan bienestar, pero también nuevos riesgos y externalidades negativas. No obstante, dependemos de ellos y del petróleo, que es la base energética vertebral global.

El otro aspecto en común es que estas empresas o industrias privilegian la calidad del recurso humano como factor de competitividad sostenible y dinámica. El ejemplo de Microsoft ejemplifica bien esta afirmación. Esta empresa sostiene que el capital humano es la principal fuente de ventaja competitiva y, por tanto, todos los trabajadores son altamente creativos, inconformes con el estado actual de cosas, comprometidos con la empresa y la sociedad para la cual diseñan sus productos. No es extraño oír a sus principales directivos realizar afirmaciones como: «El principal requisito para formar parte de la famita Microsoft es ser una persona de banda ancha».

La gestión del conocimiento ha permitido, sin duda, correlacionar los intangibles con valor de mercado con aquellos cuyas externalidades positivas y/o negativas aún no han sido valorizadas, tal como ocurre con los nuevos rankings empresariales que incluyen el criterio de responsabilidad social. Pero, nuevamente, esta posibilidad parece lejana o ajena al contexto de negocios de las pequeñas empresas, en particular en las economías en vías de desarrollo de la región. No obstante, se ha encontrado en pequeñas empresas en el Perú que siguen este credo evidencias de que la búsqueda de un mayor conocimiento y su aprovechamiento para lograr una mejor conexión con las necesidades y los desafíos de la sociedad explica su crecimiento, aumento de productividad, competitividad y éxito económico.

\section{La formación de redes de cooperación}

Independientemente de su tamaño, las empresas exitosas son aquellas que siguen el principio de competir y cooperar al mismo tiempo. Alianzas, redes, asociaciones $\mathrm{y}$ joint-ventures las ayudan a emprender nuevos rumbos y disminuir los riesgos de aventurarse solos o confiar únicamente en sus capacidades individuales. Estas compañías demuestran que es posible despojarse del celo sobre sus procesos, tecnologías y estrategias para ponerlos al servicio de potenciales aliados, quienes a su vez harán lo mismo.

Así, por ejemplo, es común encontrar que empresas que compiten de manera encarnizada en un determinado rubro de productos son aliadas en el desarrollo de nuevas tecnologías y diversas aplicaciones para esas tecnologías. Este es un caso común en grandes empresas como IBM que durante años ha competido con HewlettPackard por el predominio en el desarrollo y la comercialización de computadoras de escritorio, servidores y soluciones corporativas; pero, al mismo tiempo, ha colaborado en forma activa con esta empresa para desarrollar nuevos tipos de productos y sistemas informáticos para su uso en redes computacionales. Este mismo patrón de cooperación ha sido hallado en pequeñas empresas manufactureras en el Perú.

El caso de la empresa Acrimetal, que opera en el sector metal-mecánico, es ilustrativo. Acrimetal compite en el segmento de productos de metal y fibra de vidrio de costo medio con un gran número de microempresas que ofrecen productos similares a precios bastante menores. Algunas de esas empresas son conducidas por antiguos trabajadores que decidieron emprender su propio negocio luego de haber 
aprendido el arte de trabajar con fibra de vidrio y metal. Pero Acrimetal, a pesar de enfrentar la nueva competencia por precios, ha decidido trabajar con algunos de sus pequeños competidores que ofrecen una mejor calidad.

El acuerdo comprende la transferencia de métodos de manufactura a cambio de producir para Acrimetal con igual calidad a la que brindan a sus clientes. Este acuerdo de subcontratación y alianza ha demostrado ser una estrategia de tipo simbiótico que ha incrementado las utilidades de Acrimetal y las de las empresas «satélite», como las denomina el propietario de Acrimetal, y mejorado su posicionamiento en el mercado.

\section{El desarrollo del sentido de ser buen ciudadano}

Las pequeñas empresas exitosas también han emulado a sus pares en el segmento de la mediana y la gran empresa en el sentido de adoptar una mayor conciencia con relación al impacto social, ambiental y en la salud asociado a sus actividades. Han comprendido que disponen de los recursos, el conocimiento, la influencia $\mathrm{y}$, en general, los medios para lograr que su actividad tenga un menor impacto sobre el ambiente, emplear las materias primas de manera más eficiente, mantener las tradiciones de sus localidades y colaborar con iniciativas sociales. Esto puede que signifique un buen márketing de imagen pero el conjunto de criterios para desarrollar un sentido de ciudadanía que las guía no está orientado por el ánimo de lucro.

Estas empresas han tomado debida cuenta de que sus impuestos no son más que una renta justa por el uso de los recursos que explotan o el espacio que ocupan y que su real sentido de responsabilidad social está en llevar a cabo iniciativas que contribuyan al desarrollo regional o local. El efecto directo de este sentido de solidaridad ha sido que la sociedad las reconozca como «buenos vecinos»y, en muchos casos, sus productos son preferidos en mercados que valoran el sentido de ciudadanía de las empresas.

\section{El sentido de equidad: uno más uno es igual a «uno»}

Al revisar los tres elementos de la responsabilidad social previamente identificados $\mathrm{y}$ descritos se puede pensar que se trata de factores que pueden ser mejor aprovechados por las grandes empresas o las corporaciones. No obstante, se plantea que estos elementos son relevantes dentro de la fórmula de competitividad interna que las pequeñas empresas deben desarrollar para alcanzar y sostener su crecimiento y prestigio.

Las pequeñas empresas que en la actualidad destacan por tener un crecimiento saludable parecen ser aquellas que desarrollan la habilidad de gestionar el conocimiento, impulsar la innovación, fortalecer las capacidades de sus trabajadores y atraer empleados más creativos y comprometidos. Lideran o apoyan a movimientos de asociatividad, agremiación y reunión entre sus pares bajo el criterio de que la eficiencia individual en el estrato de la pequeña empresa es mucho menor que el nivel de eficiencia y productividad colectivo, el cual implica la capacidad de complementar esfuerzos en beneficio de todos y ganar poder de negociación para mejorar sus costos, fijar sus precios y emprender nuevos rumbos e iniciativas de absorción de tecnologías. 
Las empresas responsables acompañan su éxito económico con un sentido de responsabilidad social en sus respectivas comunidades y son actores clave en la toma de decisiones que afectan al resto de la comunidad. Generan empleo de mejor calidad para los vecinos que trabajarán en sus instalaciones, colaboran con iniciativas sociales, culturales y de mejora del ornato, se preocupan por las condiciones de trabajo de sus empleados y la calidad de vida de sus familias. Esto significa que uno de los propósitos de toda pequeña empresa que busque competitividad sostenida consiste en asumir un nuevo papel y actitudes que la acerquen más a la realidad de su entorno local. Lo que no se consigue con la capacitación en gestión empresarial sino con la sensibilización y la difusión de experiencias de éxito en las pequeñas empresas y la adopción de un modelo de competitividad interna que incida sobre la competitividad y la productividad.

\subsection{La distribución de los resultados del negocio}

Finalmente, el tercer elemento de competitividad interna consiste en una filosofía empresarial que articula el crecimiento del negocio y el reconocimiento o la buena imagen alcanzada por la empresa con el bienestar y el acceso a oportunidades de desarrollo de sus integrantes y colaboradores. Sobre este aspecto, se puede esperar críticas en torno a la dificultad de aplicar este factor en el ámbito de las pequeñas empresas con el argumento de que solo las medianas y las grandes empresas pueden generar excedentes suficientes de manera sostenida como para asignar bonos de productividad, incrementar sueldos y salarios, y proveer beneficios sociales a sus empleados. Esta visión parcial resulta errónea, como se demostrará a continuación.
Una primera reacción en torno a la distribución de los resultados del negocio parece privilegiar la idea que sostiene que, de haber más dinero, sí se puede gratificar a los trabajadores; si esto no es así se generará un conflicto con los trabajadores quienes podrían reclamar que «lo que es de hecho también es de derecho». Es decir, si la empresa brinda un determinado beneficio o gratificación, esta debe mantenerse. Esta reacción es previsible y los empresarios pueden optar por ahorrarse una preocupación más y decidir entregar al trabajador solo el sueldo o el salario que le corresponde por su trabajo. No obstante, otras empresas asumen que la distribución del éxito económico es una inversión que permite a la empresa capitalizar su recurso más valioso: las personas. Pero esta lectura es también parcial y puede ser equivocada.

Para demostrar la necesidad de adoptar una lectura integral de la «distribución» se debe diferenciar entre «éxito empresarial»y «resultados del negocio». El éxito empresarial se obtiene cuando la empresa logra posicionarse convenientemente en el mercado y abastecerlo de manera permanente, con lo cual obtiene flujos de caja positivos y relativamente constantes*. En cambio «los resultados del negocio» suponen que inclusive cuando los flujos económicos no son positivos parte de la preocupación y las medidas de austeridad, la contracción de la actividad empresarial $u$ otros similares deben ser compartidos con trabajadores y empleados. Estos no estarán en principio «motivados» para plantear que sus sueldos o salarios sean rebajados porque en principio no son ellos quienes toman las decisiones estratégicas de nivel

* No se debe olvidar el factor estacional por el cual no todos los niveles de ventas se pueden mantener estables durante el año. Hay meses de alta demanda y otros de baja demanda. 
superior cuando el poder de decisión conlleva la responsabilidad por los resultados. Si no tienen la oportunidad de contribuir a la mejor toma de decisiones, no es razonable que asuman la responsabilidad por las pérdidas. Al no haber un punto medio, una reacción casi automática por parte de empresarios y gerentes tiende a ser la reducción de personal.

Por otro lado, la teoría administrativa ha abundado en el estudio de los factores que motivan a las personas. Estas lecciones son de amplio conocimiento en el ámbito de la gran empresa, pero difícilmente lo son en el campo de acción de las pequeñas empresas en economías en vías de desarrollo. Una de las teorías que más aplicaciones ofrece para el ámbito de la pequeña empresa es la teoría de Maslow, conocida también como la pirámide de Maslow, la cual plantea que las personas experimentan cinco necesidades, tal como se muestra en el gráfico 4.

En la medida que el individuo cubra sus necesidades fisiológicas (nivel básico o primario: sed, hambre, vestido), buscará satisfacer las necesidades de seguridad (protección física, estabilidad) y así suce-

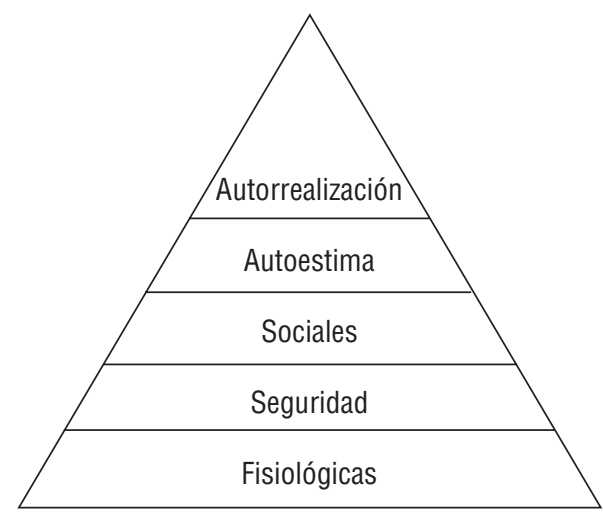

Gráfico 4

Pirámide de Maslow sivamente las del nivel siguiente: sociales o de pertenencia (contactos sociales, vida económica), autoestima o estatus (autonomía, respeto, prestigio, poder), y autorrealización (aspiraciones personales).

Si se utiliza otra de las teorías de mayor aceptación como la teoría de Herzberg también se probará que se puede apelar a factores motivacionales como bisagra entre la empresa y los trabajadores, particularmente en el ámbito de la pequeña empresa. El análisis de los dos postulados de esta teoría fácilmente llevará a concluir que también se pueden aplicar en el ámbito de las mypes. Estos factores son:

- Factores que determinan el mayor o menor grado de satisfacción en el trabajo y que están relacionados con el contenido mismo de este: aquí se encuentran elementos como la realización de un trabajo interesante, la sensación de logro, la responsabilidad y el reconocimiento.

- Factores higiénicos: generan una actitud positiva en el trabajador y derivan en un aumento de la productividad, como las condiciones de trabajo, el salario, las relaciones humanas y la política de la empresa.

Es evidente que todas o casi todas estas necesidades pueden ser cubiertas, directa o indirectamente, por la empresa. De ser así, los trabajadores entenderán que su centro laboral es como su segundo hogar, porque allí pasan gran parte de su tiempo y, por ende, le dedicarán su mejor esfuerzo y preocupación. Más aún, si existe tal grado de identificación entre los trabajadores, los empleadores contarán con una valiosa herramienta para hacer de ellos aliados en el proceso de búsqueda de resultados del 
negocio, aun en los tiempos de contracción de la demanda del mercado.

Un ejemplo claro de que es muy posible para las mypes distribuir los resultados del negocio fue identificado por los autores. En pequeñas empresas localizadas en el Parque Industrial de Villa El Salvador (Lima, Perú) y que destacan o son líderes en sus respectivos sectores de actividad se ha encontrado que existe un acuerdo tácito entre los trabajadores, los empleadores y el significado de la empresa. Los empleadores, por ejemplo, buscan transmitir a sus trabajadores lo importantes que son para el éxito y el crecimiento de la empresa y que su éxito tiene un impacto directo en la calidad de vida de sus familias. Esta buena práctica hace de estas empresas un buen lugar para trabajar.

Un ejemplo concreto se aprecia en el tipo de estrategias que siguen las pequeñas empresas de metal-mecánica y fibra de vidrio en los tiempos de baja demanda, cuando las ventas bajan, la producción decrece y existe la tentación de reducir personal. En el caso referido, el empleador implementa medidas de contingencia que todos en la empresa conocen y buscan respaldar. Una primera política es reducir la producción destinada a atender pedidos específicos para redireccionar recursos y personal hacia la producción de moldes y el desarrollo de nuevos diseños y tipos de acabado y, al mismo tiempo, producir un stock de los modelos de mayor venta que permita a la firma atender pedidos no previstos o urgentes. Una segunda política consiste en impulsar la fabricación de otros productos de menor precio (productos estándar) que pueden ser muy distintos a aquellos que integran las principales líneas de producción de la empresa; se consigue así aprovechar la capacidad instalada y mantener un ritmo sostenido de entrenamiento del personal.

Con ambas medidas se logra que la empresa mantenga un flujo de ingresos suficiente para cubrir gastos operativos y mantener «en caliente» su capacidad instalada y fuerza laboral. Asimismo, en los casos analizados se identificó que en ciclos de baja demanda los trabajadores participan en actividades que contribuyen a la mejora de las condiciones de trabajo y el mantenimiento de las instalaciones. Por ejemplo, los operarios que realizan actividades de pintura pueden emplear su destreza en labores de mantenimiento, tanto de las instalaciones de la fábrica como de equipos y maquinarias. Con esta medida no solamente se retiene a los trabajadores más valiosos, sino que se logra mantener la buena imagen de la empresa ante los ojos de clientes y visitantes. Esto significa que la pequeña empresa que aprovecha los elementos de competitividad interna adopta fórmulas para compartir con sus trabajadores aquellas acciones que favorecen a la empresa, pero también aquellos desafíos y esfuerzos requeridos para crear un mejor ambiente de trabajo, motivador y seguro, lo que estimula la mejora en la productividad.

\section{Conclusiones y expectativas}

La competitividad de la pequeña empresa ha cobrado mayor relevancia entre las estrategias de desarrollo porque los emprendimientos asociados a este estrato empresarial son pieza clave de los modelos económicos en la región Asia Pacífico y, especialmente, en las economías en vías de desarrollo. Consecuentemente, en 
las últimas dos décadas los gobiernos han asignado mayores recursos a programas de fomento y servicios de desarrollo empresarial orientados a la pequeña empresa.

En particular, las economías en vías de desarrollo también han articulado su modelo económico nacional con iniciativas de desarrollo económico local, muchas de las cuales buscan añadir impulso al crecimiento y la competitividad de la pequeña empresa mediante políticas e intervenciones externas, canalizadas a través de los gobiernos subnacionales. Estas intervenciones, en el cual el control lo tiene el agente promotor, han sido de dos tipos. El primer tipo de intervenciones externas comprende inversión en infraestructura, microfinanciamiento y comunicaciones, mientras el segundo tipo de intervenciones externas consisten en programas de apoyo asociados a capacitación y asistencia técnica en el empleo de tecnologías para la gestión, entre otros.

No obstante, los estudios realizados sugieren que las iniciativas externas de promoción al emprendimiento y la pequeña empresa no han tenido un correlato con los resultados en términos de crecimiento; en efecto, los indicadores asociados a la competitividad y la productividad (consensuados en diversos estudios consultados) han reflejado rendimientos menores a los que cabría esperar en función de las inversiones realizadas por los entes promotores gubernamentales.

Se propone que la brecha en cuanto a los resultados esperados obedece a dos aspectos. El primero alude al hecho que los programas de fomento emprendedor logran resultados sostenibles en la medida en que se cuenta con el compromiso de los emprendedores locales. A la luz de los hallazgos en los principales clusters de pequeñas empresas en el Perú y de la bibliografía consultada, se plantea que por parte de los emprendedores locales existe una postura bastante más receptiva hacia el apoyo externo en comparación con asumir compromisos sustantivos para mejorar la productividad y la competitividad. Sobre dicha base, el segundo aspecto alude a la falsa percepción de que la magnitud del esfuerzo interno para aumentar la productividad y la competitividad sostenida solamente está al alcance de las medianas y grandes empresas, lo que refuerza la postura más receptiva e inhibe de mayores compromisos.

En tal sentido, se estima que si bien la importancia de la pequeña empresa justifica la formulación e implementación de iniciativas de desarrollo económico local que la incluyan, es necesario complementar dichas intervenciones externas con estrategias internas para el crecimiento y la competitividad sostenida. Se requerirá, por tanto, de emprendimientos internos que tengan un impacto sustantivo en las mejoras de productividad y competitividad. Estas estrategias han sido sistematizadas y presentadas en un modelo de competitividad sostenible de la pequeña empresa en el que convergen la innovación de procesos, productos y tecnologías de gestión, el manejo y la motivación del capital humano, la responsabilidad empresarial frente a los desafíos del desarrollo local, la cooperación interempresarial y la articulación de acciones con el gobierno local, entre otras.

El modelo articula intervenciones externas (de organismos gubernamentales y, cada vez más, de organizaciones privadas) y esfuerzos internos para innovar políticas $\mathrm{y}$ estrategias que puedan sostenerse en el 
tiempo y recompensar a los verdaderos emprendedores. A la vez, constituye un marco de referencia que se espera contribuya a la evolución de los acuerdos tácitos entre los organismos que apoyan a la pequeña empresa (públicos y privados) y los emprendedores locales con relación a reemplazar el enfoque asistencialista por un acuerdo cooperativo que institucionalice aquellas políticas que encuentran correlato en la evolución de la pequeña empresa, en términos de crecimiento sostenido y competitividad sostenible, aspectos que aluden a indicadores que reflejan el papel de la pequeña empresa en la creación de prosperidad y ventaja competitiva, tal como viene sucediendo en las economías más pujantes del Asia Pacífico y en países europeos que, como Finlandia e Irlanda del Norte, han tenido gran éxito en ampliar su competitividad nacional sobre la base de la pequeña empresa.

\section{Referencias bibliográficas}

CANTWELL, J. 2004. Innovation and competitiveness. En Fagerberg, Jan; Mowery, David C. y Nelson, Richard R. (eds.). The Oxford handbook of innovation. New York: Oxford University, págs. 543-568.

CHRISMAN, J. y MCMULLAN, W. (eds.). 2002. Some additional comments on the sources and measurement of the benefits of small business assistance programs. Journal of Small Business Management (University of Calgary). Alberta, vol. 40, n. ${ }^{\circ} 1$, págs. 43-50.

CHRISTENSEN, C. M. 1997. The innovator's dilemma: When new technologies cause great firms to fail. Boston: Harvard Business School.

FISCHER, E. y REUBER, R. 2003. Industrial clusters and business development services for small and medium-sized enterprises. En Ganeshan, Wignaraja (eds.). Competitiveness strategy in developing countries: A manual for policy analysis. London: Routedge, págs. 131-165.

HIGH, J. 2004. The roles of entrepreneurship in economic growth: Toward a theory of total factor productivity. En De Groot, Henri; Nijkamp, Peter y Stough, Roger R. (eds.).
Entrepreneurship and regional economic development. Cheltenham, UK: Edward Elgar, págs. 46-77.

HOMMEN, L. y EDQUIST, C. 2008. Globalization and innovation policy. En Edquist, Charles y Hommen, Leif (eds.). Small country innovation systems: Globalization, change and policy in Asia and Europe. Cheltenham, UK: Edward Elgar, págs. 442-484.

INTERAMERICAN DEVELOPMENT BANK. 2002. Entrepreneurship in emerging economies: The creation and development of new firms in Latin America and East Asia. Washington D. C.: IADB Publications.

JULIEN, P. 2007. A theory of local entrepreneurship in the knowledge economy. Cheltenham, UK: Edward Elgar.

LONGENECKER, J. G.; MOORE, C. W. y PETTY, J. W. 2001. Administración de pequeñas empresas: un enfoque emprendedor. México D. F.: Thomson Internacional.

NONAKA, I. y TAKEUCHI, H. 1995. The knowledge-creating company: How Japanese firms create the dynamics of innovation. Oxford: Oxford University. 
PAGES, E.; FREEDMAN, D. y VON BARGEN, P. 2003. Entrepreneurship as a state and local economic development strategy. En Hart, David M. (ed.). The emergence of entrepreneurship policy. New York: Cambridge University, págs. 240-259.

PARKER, S. 2004. The economics of selfemployment and entrepreneurship. New York: Cambridge University Press.

PITELIS, C. y PSEIRIDIS, A. 2006. A conceptual framework for firm cooperation and clusters, and their impact on productivity. En Pitelis, Christos; Sugden, Roger y Wilson, James R. (eds.). Clusters and globalization: the development of urban and regional economies. Cheltenham, UK: Edward Elgar, págs. 17-60

ROCA, S. y VARGAS, B. 2003. Pequeña empresa: estrategias sistémicas para el crecimiento en un entorno global. Perspectiva Econômica (Universidade Do Vale Do Rio Dos Sinos). São Leopoldo, RS., vol. 7, n. ${ }^{\circ}$ 12 , págs. 65-82.
RUTTEN, R. y BOEKEMA, F. 2004. A knowledge-based view on innovation in regional networks: The case of the KIC project. En De Groot, Henri; Nijkamp, Peter y Stough, Roger R. (eds.). Entrepreneurship and regional economic development. Cheltenham: Edward Elgar. págs. 175-197.

SERIDA, J. y otros. 2005. Global Entrepreneurship Monitor. Perú 2004-2005. Lima: ESAN.

SCHUMPETER, J. A. 1984. Capitalismo, socialismo y democracia. Barcelona: Orbis.

VARGAS, B. 2007. Políticas de fomento a la competitividad de la pequeña empresa. Cuadernos de Difusión (ESAN). Lima, jun., vol. 12 , n. ${ }^{\circ} 22$, págs. $35-63$. 